\title{
CONDITIONAL WEGNER ESTIMATE FOR THE STANDARD RANDOM BREATHER POTENTIAL
}

\author{
MATTHIAS TÄUFER AND IVAN VESELIĆ
}

\begin{abstract}
We prove a conditional Wegner estimate for Schrödinger operators with random potentials of breather type. More precisely, we reduce the proof of the Wegner estimate to a scale free unique continuation principle. The relevance of such unique continuation principles has been emphasized in previous papers, in particular in recent years.

We consider the standard breather model, meaning that the single site potential is the characteristic function of a ball or a cube. While our methods work for a substantially larger class of random breather potentials, we discuss in this particular paper only the standard model in order to make the arguments and ideas easily accessible.
\end{abstract}

\section{INTRODUCTION}

A Wegner estimate is an upper bound on the expected number of eigenvalues in a prescribed energy interval of a finite box Hamiltonian. The expectation here refers to the potential which is random. Wegner estimates have been derived for Hamiltonians living on $\mathbb{Z}^{d}$ or $\mathbb{R}^{d}$, more precisely on bounded subsets of rectangular shape of these spaces. In the present note we do not put emphasis on presenting the history of various variants of the Wegner estimate, but rather refer to the monograph [18 and recent papers [14, and 9]. Here we will be only interested in models on continuum space $\mathbb{R}^{d}$. The most studied example in this situation is the so called alloy-type potential, sometimes also called continuum Anderson model. A particular feature of this model is that randomness enters the model via a countable number of random variables, and these r.v. influence the potential in a linear way. In the model we study here this dependence is no longer linear, but becomes non-linear. What remains, is the monotone dependence of the potential on the r.v. The topic of the present note is to explain, how to effectively use this monotonicity. This only works if it is possible to cast the monotonicity in a quantitative form. With this respect we consider the study of the random breather model as paradigmatic for a better understanding of random Schrödinger operators with non-linear randomness.

Key words and phrases. random potential, standard random breather potential, Schrödinger operators, Wegner estimate,

(C) 2014 by the authors. Faithful reproduction of this article, is permitted for noncommercial purposes. July 21, 2018, 2015-09-08standardRB.tex.

This work has been partially supported by the DFG under grant Eindeutige Fortsetzungsprinzipien und Gleichverteilungseigenschaften von Eigenfunktionen. It has also profited from interactions with Francisco Hoecker-Escuti, Ivica Nakić, Martin Tautenhahn and Christoph Schumacher. Part of these interactions have been supported by the binational German-Croatian DAAD project Scale-uniform controllability of partial differential equations. 
Note that in the case that one aims to establish a Wegner estimate only in an energy region near the bottom of the spectrum, it is possible to overcome the monotonicity assumption. A paradigmatic example is the random displacement model, cf. 10. However let us clarify that any Wegner estimate proven so far uses at least one of the following properties

(a) The space dimension is one.

(b) The random potential has a quantitative monotonicity property.

(c) One restricts attention to an energy region near the spectral bottom (or some other spectral edge, however this requires again extra assumptions, like small disorder).

A strategy to prove Wegner estimates without relying on any of the above properties seems to require a truly probabilistic approach, rather than an analyitic one, like averaging just over a finite number of random variables.

To our best knowledge random breather potentials have been first considered in the mathematical physics literature in the work 2. A Wegner estimate for the random breather model was derived in [3] and a Lifschitz tail bound, yielding localization, in 8 . However, all these papers have a condition on the gradient of the single site potential, arising from a linearization. Unfortunately, this excludes the most elementary single site potential, namely the characteristic function of a ball or a cube. A simple situation where this was overcome is treated in [17] where a Lifschitz bound was proven. Actually this proof extends to very general breather models, as will be explained in [15].

The aim of this note is not to cover the most general types of random breather potentials, but to concentrate on the simplest case and provide full proofs and calculations accessible to non-specialists. For this purpose we spell out explicitly the theorems which we infer, rather than just giving references to earlier papers. Our proof starts from a scale free unique continuation principle (SFUCP). Such an estimate has recently been proven, for the case that the magnetic vector potential vanishes. It is announced in [13 and full proofs will be presented in [12], which rely on Carleman estimates, interpolation inequalities and related PDE techniques. The proofs in this note are more probabilistic in nature.

1.1. Wegner estimate for the random breather model. We prove a Wegner estimate, Theorem 1.3. for the Random Breather model. In the following, $\Lambda_{s}(x):=x+(-s / 2, s / 2)^{d}$ is the $d$-dimensional open cube of side length $s>0$, centered at the point $x \in \mathbb{R}^{d}$. $B_{r}(x)$ denotes the open ball of radius $r \geqslant 0$ around $x \in \mathbb{R}^{d}$. If $x=0$, we omit the $x$ and write $\Lambda_{s}$ or $B_{r}$. If the side length $s$ is fixed, we simply write $\Lambda$. Let $0 \leqslant \omega_{-}<\omega_{+}<1 / 2$ and let $\mu$ be a probability measure on $\mathbb{R}$ with bounded density $\nu_{\mu}$ and support in $\left[\omega_{-}, \omega_{+}\right]$. We define the probability space

$$
(\Omega, \mathcal{A}, \mathbb{P})=\left(\times_{i \in \mathbb{Z}^{d}} \mathbb{R}, \otimes_{i \in \mathbb{Z}^{d}} \mathcal{B}(\mathbb{R}), \otimes_{i \in \mathbb{Z}^{d}} \mu\right) .
$$

Here, $\mathcal{B}$ is the Borel $\sigma$-algebra. For $\omega \in \Omega$ and $j \in \mathbb{Z}^{d}$ we denote the projection onto the $j$-th coordinate of $\Omega$ by $\omega_{j}$. The $\left\{\omega_{j}\right\}_{j \in \mathbb{Z}^{d}}$ form a process of $\left[\omega_{-}, \omega_{+}\right]$valued independent and identically distributed random variables on $\mathbb{Z}^{d}$. For 
$i, j \in \mathbb{Z}^{d}$ and $\delta \in \mathbb{R}$ we define $\omega+\delta$ and $\omega+\delta e_{i} \in \Omega$ by

$$
\begin{array}{r}
(\omega+\delta)_{j}:=\omega_{j}+\delta \text { for all } j \in \mathbb{Z}^{d} \\
\left(\omega+\delta e_{i}\right)_{j}:= \begin{cases}\omega_{j}+\delta & \text { if } j=i \\
\omega_{j} & \text { if } j \neq i .\end{cases}
\end{array}
$$

Definition 1.1. We consider a nonnegative and self-adjoint magnetic Schrödinger operator with vector potential $A \in L_{\text {loc }}^{2}\left(\mathbb{R}^{d}, \mathbb{R}^{d}\right)$

$$
H_{A}:=(-i \nabla-A)^{2}
$$

on $L^{2}\left(\mathbb{R}^{d}\right)$. We define a random potential by

$$
\begin{aligned}
& V_{\omega}(x):=\sum_{j \in \mathbb{Z}^{d}} \chi_{B_{\omega_{j}}}(x-j) \text { or } \\
& V_{\omega}(x):=\sum_{j \in \mathbb{Z}^{d}} \chi_{\Lambda_{2 \omega_{j}}}(x-j)
\end{aligned}
$$

and define the corresponding random operator $H_{\omega}:=H_{A}+V_{\omega}$. In both cases we call $H_{\omega}$ the standard random breather model. For $L \in \mathbb{N}_{\text {odd }}=\{1,3, \ldots\}$ we define the restriction $H_{\omega, L}$ of $H_{\omega}$ to $\Lambda_{L}$ with Dirichlet boundary conditions and the restriction of the potential $V_{\omega, L}: \Lambda_{L} \rightarrow \mathbb{R}$.

Definition 1.2. Let $\delta \in(0,1 / 2)$. A sequence $\left\{x_{j}\right\}_{j \in \mathbb{Z}^{d}}$ is called $\delta$-equidistributed if for every $j \in \mathbb{Z}^{d}$ we have $B_{\delta}\left(x_{j}\right) \subset \Lambda_{1}(j)$. For such a sequence and $L \in \mathbb{N}_{\text {odd }}$, we define $W_{\delta, L}: \Lambda_{L} \rightarrow \mathbb{C}$ as the characteristic function of $\bigcup_{j \in \mathbb{Z}^{d}} B_{\delta}\left(x_{j}\right) \cap \Lambda_{L}$.

We formulate a scale free quantitative unique continuation property, which the random operator $H_{\omega}$ may or may not have. Denote by $\chi_{I}\left(H_{\omega, L}\right)$ the spectral projector of $H_{\omega, L}$ onto an interval $I$.

Hypothesis (SFUCP). Given $b \in \mathbb{R}$ there are $M \geqslant 1, \kappa \leqslant 1$ such that for all $L \in \mathbb{N}_{\text {odd }}$, almost all $\omega \in \Omega$ and for all $0<\delta<1 / 2-\omega_{+}$we have

$$
\chi_{(-\infty, b]}\left(H_{\omega, L}\right) W_{\delta, L} \chi_{(-\infty, b]}\left(H_{\omega, L}\right) \geqslant \kappa \delta^{1 / M} \chi_{(-\infty, b]}\left(H_{\omega, L}\right) .
$$

Inequality (3) is understood in the sense of quadratic forms.

Theorem 1.3 (Wegner estimate for the Random Breather model). Assume that the standard random breather model satisfies hypothesis SFUCP. Let $b \in \mathbb{R}$. Then there are constants $C=C(d, b) \in(0, \infty), \varepsilon_{\max }=\varepsilon_{\max }\left(\kappa, M, \omega_{+}\right) \in(0, \infty)$ such that for all $L \in \mathbb{N}_{\text {odd }}$, all $0<\varepsilon \leqslant \varepsilon_{\max }, E \in \mathbb{R}$ with $[E-\varepsilon, E+\varepsilon] \subseteq$ $(-\infty, b-1]$ we have

$$
\mathbb{E}\left[\operatorname{Tr}\left[\chi_{[E-\varepsilon, E+\varepsilon]}\left(H_{\omega, L}\right)\right]\right] \leqslant C \sqrt[M]{4 \kappa^{-1}}\left\|\nu_{\mu}\right\|_{\infty} \varepsilon^{1 / M}|\ln \varepsilon|^{d} L^{d} .
$$

$\varepsilon_{\max }$ can be chosen as

$$
\varepsilon_{\max }=\frac{\kappa}{4}\left(\frac{1 / 2-\omega_{+}}{2}\right)^{M} .
$$

Remark 1.4. (i) We can give an explicit bound on the constant $C$, namely

$$
C \leqslant 2 \cdot 32^{d}\left(2 e^{b} \cdot(d+1) !+2^{d}\right) .
$$


(ii) The $|\ln \varepsilon|^{d}$ term can be hidden by choosing a slightly smaller $\tilde{M}<M$ and a different constant $\tilde{C}>0$. Hiding also the factor $\sqrt[M]{4 \kappa^{-1}}\left\|\nu_{\mu}\right\|_{\infty}$ in this constant, we obtain a bound of the form

$$
\mathbb{E}\left[\operatorname{Tr}\left[\chi_{[E-\varepsilon, E+\varepsilon]}\left(H_{\omega, L}\right)\right]\right] \leqslant \tilde{C} \cdot \varepsilon^{1 / \tilde{M}} L^{d} .
$$

This implies local Hoelder continutity of the integrated density of states with the exponent $1 / \tilde{M}$ as in [5]

(iii) If SFUCP only holds for $L \in \mathcal{I} \subset \mathbb{N}_{\text {odd }}$ then Theorem 1.3 holds for $L \in \mathcal{I}$.

\section{Estimates on the SPECTRAL Shift FUnCtion}

We infer here the estimates on the singular values of semingroup differences and the spectral shift function of two Schrödinger operators differing by a compactly supported potential obtained in [5]. We reduce the estimates to the particular, simple situation we are dealing with. The constants are calculated explicitly and more accurately than in [5. We will be dealing here with a pair of operators: $H_{0}=H_{A}+V_{0}$ with $V_{0}$ non-negative and bounded, and $H_{1}:=H_{0}+V$, with $V$ non-negative, bounded, and supported in a cube $\Lambda_{1}$ of sidelength one. Let $\Lambda=\Lambda_{L}$ be a cube of sidelength $L \in \mathbb{N}_{\text {odd }}$ and call $H_{0}^{\Lambda}$ and $H_{1}^{\Lambda}$ the restrictions of $H_{0}$ and $H_{1}$ onto $\Lambda$ with Dirichlet boundary conditions. Let

$$
V_{\text {eff }}^{\Lambda}:=e^{-H_{1}^{\Lambda}}-e^{-H_{0}^{\Lambda}} .
$$

This is a compact operator and we will enumerate its singular values decreasingly by $\mu_{1} \geqslant \mu_{2} \geqslant \ldots$. Then we have the following Theorem:

Theorem 2.1 (Theorem 1 from [5]). For $n>N_{0}:=4^{d}$, the singular values of $V_{\text {eff }}^{\Lambda}$ obey

$$
\mu_{n}\left(V_{\text {eff }}^{\Lambda}\right) \leqslant(\sqrt[4]{2 d}+1) \exp \left(-\frac{n^{1 / d}}{16}\right)
$$

We start the proof with a lemma

Lemma 2.2. Let $H=H_{0}$ or $H_{1}$ be as above and let $H^{\mathcal{U}}$ be the Dirichlet restriction of $H$ to an open set $\mathcal{U}$ with finite volume $|\mathcal{U}|$. Then the $n^{\text {th }}$ eigenvalue $E_{n}$ of $H^{\mathcal{U}}$ satisfies

$$
E_{n} \geqslant \frac{2 \pi d}{e}\left(\frac{n}{|\mathcal{U}|}\right)^{2 / d} .
$$

Proof. We have $H^{U} \geqslant H_{A}^{\mathcal{U}}$ where $H_{A}^{\mathcal{U}}$ is the Dirichlet restriction of $H_{A}$ to $\mathcal{U}$. Hence

$$
\operatorname{Tr}\left(e^{-2 t H^{\mathcal{U}}}\right) \leqslant \operatorname{Tr}\left(e^{-2 t H_{A}^{\mathcal{U}}}\right)=\left\|e^{-t H_{A}^{\mathcal{U}}}\right\|_{\mathrm{HS}}^{2}=\iint_{\mathcal{U} \times \mathcal{U}}\left|e^{-t H_{A}^{\mathcal{U}}}(x, y)\right|^{2} \mathrm{~d} x \mathrm{~d} y
$$

where $\|\cdot\|_{\text {HS }}$ denotes the Hilbert-Schmidt norm. The diamagnetic inequality for the Schrödinger semigroup, see [7, Remark 1.2.iii] implies the pointwise bound

$$
\left|e^{-t H_{A}^{\mathcal{U}}}(x, y)\right| \leqslant e^{t \Delta^{\mathcal{U}}}(x, y) .
$$


Using that the kernel of the Dirichlet semigroup is bounded by the free kernel, that is

$$
\begin{aligned}
& \int_{\mathcal{U}}\left|e^{-t \Delta^{\mathcal{U}}}(x, y)\right|^{2} \mathrm{~d} y \leqslant \int_{\mathcal{U}}\left|e^{-t \Delta}(x, y)\right|^{2} \mathrm{~d} y=\int_{\mathcal{U}}(4 \pi t)^{-d} \exp \left(-\frac{|x-y|^{2}}{2 t}\right) \mathrm{d} y \\
& \quad \leqslant(8 \pi t)^{-d / 2} \int_{\mathbb{R}}(2 \pi t)^{-d / 2} \exp \left(-\frac{|x-y|^{2}}{2 t}\right) \mathrm{d} y=(8 \pi t)^{-d / 2},
\end{aligned}
$$

see [1] for the boundedness by the free kernel and [4] Theorem 2.3.1. for the free kernel, we estimate

$$
\left\|e^{-t H_{A}^{U}}\right\|_{\mathrm{HS}}^{2} \leqslant|\mathcal{U}|(8 \pi t)^{-d / 2}
$$

Thus

$$
\operatorname{Tr}\left(e^{-2 t H^{\mathcal{U}}}\right) \leqslant|\mathcal{U}|(8 \pi t)^{-d / 2} .
$$

Denote by $\mathcal{N}^{\mathcal{U}}(E)$ the number of eigenvalues of $H^{\mathcal{U}}$ smaller or equal to $E$. We estimate using the above bound

$$
\begin{aligned}
\mathcal{N}^{\mathcal{U}}(E) & =\int_{-\infty}^{E} \mathrm{~d} \mathcal{N}^{\mathcal{U}}(s) \leqslant e^{2 t E} \int_{-\infty}^{\infty} e^{-2 t s} \mathrm{~d} \mathcal{N}^{\mathcal{U}}(s) \\
& =e^{2 t E} \operatorname{Tr}\left(e^{-2 t H^{\mathcal{U}}}\right) \leqslant|\mathcal{U}| \cdot(8 \pi t)^{-d / 2} e^{2 t E}=|\mathcal{U}|\left(\frac{e E}{2 \pi d}\right)^{d / 2}
\end{aligned}
$$

where in the last equality $t:=\frac{d}{4 E}$ has been chosen. Since $n \leqslant \mathcal{N}^{\mathcal{U}}\left(E_{n}\right)$, this implies (5).

Proof of Theorem 2.1. The $n^{\text {th }}$ singular value will be estimated by Dirichlet decoupling at a scale $R_{n}$ which monotonously depends on $n$. Recall that $\operatorname{supp}(V) \subseteq \Lambda_{1}(x)$ for some $x \in \mathbb{R}^{d}$. Choose a large $R=R_{n}>2$ to be specified later. Call $H_{j}^{R}(j=0$ or 1$)$ the Dirichlet restriction of $H_{j}$ onto $\Lambda_{2 R}=(-R ; R)^{d}$ and let

$$
A_{R}:=e^{-H_{1}^{R}}-e^{-H_{0}^{R}} \quad \text { and } \quad D_{R}:=V_{\mathrm{eff}}^{\Lambda}-A_{R} .
$$

We apply Lemma 2.2 and find

$$
\mu_{n}\left(e^{-H_{j}^{R}}\right) \leqslant \exp \left(-\frac{\pi d}{2 e} n^{2 / d} R^{-2}\right) \leqslant \exp \left(-\frac{1}{16} n^{2 / d} R^{-2}\right)
$$

for $j=1,2$. Since $A_{R}$ is the difference of two nonnegative operators, its singular values obey the same bound:

$$
\mu_{n}\left(A_{R}\right) \leqslant \mu_{n}\left(e^{-H_{2}^{R}}\right) \leqslant \exp \left(-\frac{1}{16} n^{2 / d} R^{-2}\right) .
$$

If the operator $D_{R}$ is bounded, then $\mu_{n}\left(V_{\text {eff }}^{\Lambda}\right) \leqslant \mu_{n}\left(A_{R}\right)+\left\|D_{R}\right\|$. Our goal is therefore to estimate the norm of $D_{R}$ by using the Feynman-Kac formula for Schrödinger semigroups with Dirichlet boundary conditions, see [1] and [16]. Let $\mathbb{E}_{x}$ and $\mathbb{P}_{x}$ denote the expectation and probability for a Brownian motion 
$b_{t}$, starting at $x$. Let $\tau_{\Lambda}:=\inf \left\{t>0 \mid b_{t} \notin \Lambda\right\}$ be the exit time from $\Lambda$ and $\tau_{R}:=\inf \left\{t>0 \mid b_{t} \notin \Lambda_{2 R}\right\}$ be the exit time from $\Lambda_{2 R}$. Then

$$
\begin{aligned}
& \left(D_{R} f\right)(x)= \\
& =\mathbb{E}_{x}\left[e^{-i S_{A}(b)}\left(e^{-\int_{0}^{1}\left(V_{0}+V\right)\left(b_{s}\right) \mathrm{d} s}-e^{-\int_{0}^{1} V_{0}\left(b_{s}\right) \mathrm{d} s}\right) \chi_{\left[\tau_{\Lambda}>1\right]}(b) \chi_{\left[\tau_{R} \leqslant 1\right]}(b) f\left(b_{1}\right)\right]
\end{aligned}
$$

where $S_{A}(b)$ is the real valued stochastic process corresponding to the purely magnetic part of the Schrödinger operator. To be precise, in order to use the Feynman-Kac formula from 16 one needs $\operatorname{div} A=0$. Therefore we first choose the Coulomb gauge which implies $\operatorname{div} A=0$ and then use gauge invariance as in [11] for $A \in L_{\text {loc }}^{2}\left(\mathbb{R}^{d}, \mathbb{R}^{d}\right)$. We take the modulus whence the magnetic part drops out and using furthermore that $\chi_{\left[\tau_{\Lambda}>1\right]}(b) \leqslant 1$, we find

$$
\left|D_{R} f\right|(x) \leqslant \mathbb{E}_{x}\left[e^{-\int_{0}^{1} V_{0}\left(b_{s}\right) \mathrm{d} s} \cdot\left|e^{-\int_{0}^{1} V\left(b_{s}\right) \mathrm{d} s}-1\right| \cdot \chi_{\left[\tau_{R} \leqslant 1\right]}(b) \cdot\left|f\left(b_{1}\right)\right|\right] .
$$

Only Brownian paths which both visit $\operatorname{supp}(V)$ and leave $B_{R}$ within one unit of time contribute to the expectation. Thus, if $\tau_{V}$ is the hitting time for $\operatorname{supp}(V)$ and $\mathfrak{B}=\left\{\tau_{R} \leqslant 1, \tau_{V} \leqslant 1\right\}$, then

$$
\left|D_{R} f\right|(x) \leqslant \mathbb{E}_{x}\left[e^{-\int_{0}^{1} V_{0}\left(b_{s}\right) \mathrm{d} s} \cdot\left|e^{-\int_{0}^{1} V\left(b_{s}\right) \mathrm{d} s}-1\right| \cdot \chi_{\mathfrak{B}}(b) \cdot\left|f\left(b_{1}\right)\right|\right] .
$$

Applying Hölder's inequality yields

$$
\begin{aligned}
\left|D_{n} f\right|(x) & \leqslant\left(\mathbb{E}_{x}\left[e^{-8 \int_{0}^{1} V_{0}\left(b_{s}\right) \mathrm{d} s}\right]\right)^{1 / 8}\left(\mathbb{E}_{x}\left[\left|e^{-\int_{0}^{1} V\left(b_{s}\right) \mathrm{d} s}-1\right|^{8}\right]\right)^{1 / 8} \\
& \times\left(\mathbb{E}_{x}\left[\chi_{\mathfrak{B}}(b)\right]\right)^{1 / 4}\left(\mathbb{E}_{x}\left[\left|f\left(b_{1}\right)\right|^{2}\right]\right)^{1 / 2} .
\end{aligned}
$$

Since $V, V_{0} \geqslant 0$, the first two terms are bounded by 1 .

Next, we estimate $\mathbb{E}_{x}\left[\chi_{\mathfrak{B}}(b)\right]=\mathbb{P}_{x}(\mathfrak{B})$. Letting $b_{t}=\left(b_{t}^{(1)}, \ldots, b_{t}^{(n)}\right) \in \mathbb{R}^{d}$ and calling $\tau_{r}^{(j)}:=\inf \left\{t>0 \mid b_{t}^{(j)} \notin(-r, r)\right\}$ the exit time of the $j^{\text {th }}$ coordinate from the interval $(-r, r)$, where we choose $r:=\operatorname{dist}\left\{\operatorname{supp} V, \Lambda_{2 R}^{c}\right\} \geqslant 3 / 2$, we have

$$
\mathbb{P}_{0}\left[\tau_{r} \leqslant 1\right]=\mathbb{P}_{0}\left[\bigcup_{j=1}^{d}\left\{\tau_{r}^{(j)} \leqslant 1\right\}\right] \leqslant \sum_{j=1}^{d} \mathbb{P}_{0}\left[\tau_{r}^{(j)} \leqslant 1\right]=d \cdot \mathbb{P}_{0}\left[\tau_{r}^{(1)} \leqslant 1\right] .
$$

The projection onto the first coordinate of $\left(b_{t}\right)$ is a one-dimensional Brownian motion and by the reflection principle

$$
\mathbb{P}_{0}\left[\tau_{r}^{(1)} \leqslant 1\right]=2 \mathbb{P}_{0}\left[\mid b_{1}^{(1)} \geqslant r\right]=\frac{4}{\sqrt{2 \pi}} \int_{r}^{\infty} e^{-x^{2} / 2} \mathrm{~d} x \leqslant \frac{4}{\sqrt{2 \pi}} r^{-1} e^{-r^{2} / 2} .
$$

Recalling that $r>3 / 2$, and hence $\frac{4}{\sqrt{2 \pi}} r^{-1} \leqslant 2$ we find

$$
\mathbb{P}_{0}\left[\tau_{r} \leqslant 1\right] \leqslant 2 d \cdot e^{-r^{2} / 2} .
$$

Since every path in $\mathfrak{B}$ must cover the distance $r \geqslant 3 / 2$ between $\operatorname{supp}(V)$ and the complement of $\Lambda_{2 R}$, we find $\mathbb{P}_{x}[\mathfrak{B}] \leqslant d \cdot e^{-r^{2} / 2}$. We assumed that $R>2$, hence $r \geqslant R-1 / 2 \geqslant R / \sqrt{2}$. Then $\mathbb{P}_{x}[\mathfrak{B}] \leqslant d e^{-R^{2} / 4}$ and

$$
\left|D_{R} f\right|(x) \leqslant \sqrt[4]{2 d} \cdot e^{-R^{2} / 16}\left(\mathbb{E}_{x}\left|f\left(b_{1}\right)\right|^{2}\right)^{1 / 2}=\sqrt[4]{2 d} \cdot e^{-R^{2} / 16}\left(\left(e^{\Delta}|f|^{2}\right)(x)\right)^{1 / 2} .
$$


Using the fact, that $e^{\Delta}$ is an $L^{1}$ contraction

$\left\|D_{n} f\right\|_{2} \leqslant \sqrt[4]{2 d} \cdot e^{-R^{2} / 16}\left\|\left(e^{\Delta}|f|^{2}\right)\right\|_{1}^{1 / 2} \leqslant \sqrt[4]{2 d} \cdot e^{-R^{2} / 16}\left\|f^{2}\right\|_{1}^{1 / 2}=\sqrt[4]{2 d} \cdot e^{-R^{2} / 16}\|f\|_{2}$.

To balance between the two bounds obtained for $\mu_{n}\left(A_{R}\right)$ and $\left\|D_{R}\right\|$, we choose $R:=n^{1 / 2 d}$ and find

$$
\begin{aligned}
\mu_{n}\left(A_{R}\right) & \leqslant \exp \left(-1 / 16 \cdot n^{1 / d}\right), \\
\left\|D_{n}\right\| & \leqslant \sqrt[4]{2 d} \cdot \exp \left(-1 / 16 \cdot n^{1 / d}\right),
\end{aligned}
$$

that is

$$
\mu_{n}\left(V_{\text {eff }}^{\Lambda}\right) \leqslant(\sqrt[4]{2 d}+1) \cdot \exp \left(-1 / 16 n^{1 / d}\right) .
$$

We assumed $R=n^{1 / 2 d}>2$, thus this only works for

$$
n>N_{0}=4^{d} \text {. }
$$

Let now $g \in C^{\infty}(\mathbb{R})$ with compactly supported derivative. If $g\left(H_{1}\right)-g\left(H_{0}\right)$ is trace class then there is a unique function $\xi\left(\lambda, H_{1}, H_{0}\right)$ called the Lifshitz-Krein spectral shift function, such that

$$
\operatorname{Tr}\left[g\left(H_{1}\right)-g\left(H_{0}\right)\right]=\int \xi\left(\lambda, H_{1}, H_{0}\right) \mathrm{d} g(\lambda) .
$$

This is referred to as Krein's trace identity. $\xi\left(\cdot, H_{1}, H_{0}\right)$ is independent of the choice of $g$. In fact, $g$ can be chosen from a substantially larger class of functions:

Proposition 2.3 (Chapter 8.9, Theorem 1 in [19]). Let $H_{0}, H_{1}$ be positive definite and $H_{1}^{-\tau}-H_{0}^{-\tau}$ trace class for some $\tau>0$. Furthermore let $g$ have two locally bounded derivatives and satisfy

$$
\left|\left(\lambda^{\tau+1} g^{\prime}(\lambda)\right)^{\prime}\right| \leqslant C \lambda^{-1-\varepsilon} \text {, as } \lambda \rightarrow \infty
$$

for some $C, \varepsilon>0$. Then (7) holds for $g$.

For such admissible functions, it is possible to make a change of variables

$$
\xi\left(\lambda, H_{1}, H_{0}\right)=\operatorname{sgn}\left(g^{\prime}\right) \xi\left(g\left(\lambda, g\left(H_{1}\right), g\left(H_{0}\right)\right),\right.
$$

see [19], Chapter 8.11. This is referred to as the invariance principle for the spectral shift function. With our choice of $H_{0}$ and $H_{1}, g(\lambda):=\exp (-\lambda)$ is an admissible function, as can be seen via Lemma 2.2. We also define functions $F_{t}:[0, \infty) \rightarrow[0, \infty)$ for $t>0$ by

$$
F_{t}(x):=\int_{0}^{x}\left(\exp \left(t y^{1 / d}\right)-1\right) \mathrm{d} y
$$

Theorem 2.4 (Theorem 2 from [5]). Let $\xi$ be the spectral shift function for the pair of operators $H_{0}^{\Lambda}$ and $H_{1}^{\Lambda}$.

(i) There is a constant $K_{1}$, depending on $t$ such that for small enough $t>0$

$$
\int_{-\infty}^{T} F_{t}\left(\mid \xi(\lambda \mid) d \lambda \leqslant K_{1} e^{T}<\infty .\right.
$$

$t$ can be chosen to be $t:=1 / 32$ in which case $K_{1} \leqslant 2 \cdot 32^{d} \cdot(d+1)$ ! 
(ii) There are constants $K_{1}, K_{2}$, only depending ond such that for any bounded function $f$ with compact support within $(-\infty, b]$ we have

$$
\int f(\lambda) \xi(\lambda) \mathrm{d} \lambda \leqslant K_{1} e^{b}+K_{2}\left(\log \left(1+\|f\|_{\infty}\right)\right)^{d}\|f\|_{1} .
$$

We may choose $K_{1}:=2 \cdot 32^{d}(d+1)$ ! and $K_{2}:=32^{d}$.

Corollary 2.5. Let $g \in C^{\infty}(\mathbb{R})$ with $g^{\prime}$ of compact support within $(-\infty, b]$ such that $g\left(H_{1}^{\Lambda}\right)-g\left(H_{0}^{\Lambda}\right)$ is trace class. Then

$$
\operatorname{Tr}\left[g\left(H_{1}^{\Lambda}\right)-g\left(H_{0}^{\Lambda}\right)\right] \leqslant 32^{d}\left[2(d+1) ! e^{b}+\left(\log \left(1+\left\|g^{\prime}\right\|_{\infty}\right)\right)^{d}\left\|g^{\prime}\right\|_{1}\right]
$$

Proof of Theorem 2.4. (i) Using the invariance principle for the spectral shift function we have

$$
\begin{aligned}
\int_{-\infty}^{T} F_{t}\left(\left|\xi\left(\lambda, H_{1}^{\Lambda}, H_{0}^{\Lambda}\right)\right|\right) \mathrm{d} \lambda & =\int_{-\infty}^{T} F_{t}\left(\left|\xi\left(e^{-\lambda}, e^{-H_{1}^{\Lambda}}, e^{-H_{0}^{\Lambda}}\right)\right|\right) \mathrm{d} \lambda \\
& \leqslant e^{T} \int_{e^{-T}}^{\infty} F_{t}\left(\left|\xi\left(s, e^{-H_{1}^{\Lambda}}, e^{-H_{0}^{\Lambda}}\right)\right|\right) \mathrm{d} s
\end{aligned}
$$

Since the difference $V_{\text {eff }}^{\Lambda}=e^{-H_{1}^{\Lambda}}-e^{-H_{0}^{\Lambda}}$ is trace class, we can apply an estimate of [6] and find using Theorem 2.1

$$
\begin{gathered}
\int_{-\infty}^{\infty} F_{t}\left(\left|\xi\left(s, e^{-H_{1}}, e^{-H_{0}}\right)\right|\right) \mathrm{d} s \leqslant \sum_{n=1}^{\infty} \mu_{n}\left(V_{\text {eff }}\right)\left(F_{t}(n)-F_{t}(x-1)\right) \\
=\sum_{n=1}^{N_{0}} \mu_{n}\left(V_{\text {eff }}\right) \int_{n-1}^{n}\left(e^{t s^{1 / d}}-1\right) \mathrm{d} s+\sum_{n=N_{0}+1}^{\infty} \mu_{n}\left(V_{\text {eff }}\right) \int_{n-1}^{n}\left(e^{t s^{1 / d}}-1\right) \mathrm{d} s \\
\leqslant N_{0}\left(e^{t N_{0}^{1 / d}}-1\right)+(\sqrt[4]{2 d}+1) \sum_{n=1}^{\infty} e^{(t-1 / 16) n^{1 / d}} .
\end{gathered}
$$

If we choose $t$ smaller than $1 / 16$ this will be finite. We choose $t:=1 / 32$ and recall that $N_{0}=4^{d}$ so that we obtain

$$
\int_{-\infty}^{\infty} F_{t}\left(\left|\xi\left(s, e^{-H_{1}}, e^{-H_{0}}\right)\right|\right) \mathrm{d} s \leqslant 4^{d}\left(e^{1 / 8}-1\right)+(\sqrt[4]{2 d}+1) \sum_{n=1}^{\infty} e^{-1 / 32 n^{1 / d}} .
$$

To estimate the second summand we use

$\sum_{n=1}^{\infty} e^{-1 / 32 n^{1 / d}} \leqslant \int_{0}^{\infty} e^{-1 / 32 x^{1 / d}} \mathrm{~d} x=d \cdot 32^{d} \int_{0}^{\infty} e^{-y} y^{d-1} \mathrm{~d} y=d \cdot 32^{d} \Gamma(d)=d ! \cdot 32^{d}$ and find

$$
\int_{-\infty}^{\infty} F_{t}\left(\left|\xi\left(s, e^{-H_{1}}, e^{-H_{0}}\right)\right|\right) \mathrm{d} s \leqslant 4^{d}+(\sqrt[4]{2 d}+1) \cdot 32^{d} d ! \leqslant 2 \cdot 32^{d} \cdot(d+1) !
$$

To see the very last inequality we have to distinguish between $d=1$ where it can be verified directly and $d=2,3, \ldots$ in which case we use $\sqrt[4]{2 d}+1 \leqslant d+1$. 
(ii) We use Young's inequality and dualize the bound from part (i). $F_{t}$ is non-negative and convex with $F_{t}^{\prime}(0)=0$, hence its Legendre transform $G_{t}$ is well-defined and satisfies

$$
G_{t}(y):=\sup _{x \geqslant 0}\left\{x y-F_{t}(x)\right\} \leqslant y\left(\frac{\log (1+y)}{t}\right)^{d} \text { for all } y \geqslant 0 .
$$

Young's equality yields $y x \leqslant F_{t}(x)+G_{t}(y)$ and with $b:=\sup \operatorname{supp}(f)$ we find

$$
\int f(\lambda) \xi(\lambda) \mathrm{d} \lambda \leqslant \int_{-\infty}^{b} F_{t}\left(\mid \xi(\lambda \mid) \mathrm{d} \lambda+\int G_{t}(|f(\lambda)|) \mathrm{d} \lambda .\right.
$$

Using part (i), we find that the first integral is bounded by $K_{1} e^{b}$. For the second summand we estimate

$$
\int G(|f(\lambda)|) \mathrm{d} \lambda \leqslant \int|f(\lambda)|\left(\frac{\log (1+|f(\lambda)|)}{t}\right)^{d} \mathrm{~d} \lambda \leqslant t^{-d} \mid \log \left(1+\|f\|_{\infty}\right)^{d}\|f\|_{1} .
$$

\section{Proof of the Wegner estimate}

We will occationally write $H_{L}(\omega)=H_{\omega, L}$ and $V_{L}(\omega)=V_{\omega, L}$ for notational convenience. Note that for all $\omega \in\left[\omega_{-}, \omega_{+}\right]^{\mathbb{Z}^{d}}$, all $L \in \mathbb{N}_{\text {odd }}$ and all $j \in \mathbb{Z}^{d}, H_{\omega, L}$ has purely discrete spectrum. Denote the eigenvalues of $H_{\omega, L}$ by $\left\{\lambda_{i}(\omega)\right\}_{i \in \mathbb{N}}$, enumerated increasingly and counting multiplicities.

Lemma 3.1. Let (3) hold and assume that $\omega \in\left[\omega_{-}, \omega_{+}\right]^{\mathbb{Z}^{d}}$. Then for all $i \in \mathbb{N}$ with $\lambda_{i}(\omega) \in(-\infty, b-1]$ and all $\delta \leqslant 1 / 2-\omega_{+}$we have

$$
\lambda_{i}(\omega+\delta) \geqslant \lambda_{i}(\omega)+\kappa(\delta / 2)^{1 / M} .
$$

Proof. Let $H_{\omega+\delta, L} \phi_{i}=\lambda_{i}(\omega+\delta) \phi_{i}$ for all $i \in \mathbb{N}$. Then

$$
\lambda_{i}(\omega+\delta)=\left\langle\phi_{i}, H_{\omega+\delta, L} \phi_{i}\right\rangle .
$$

We write $H_{\omega+\delta, L}=H_{\omega, L}+\left(V_{\omega+\delta, L}-V_{\omega, L}\right)$. Note that $\omega_{+}+\delta \leqslant 1 / 2$ and

$$
V_{\omega+\delta, L}(x)-V_{\omega, L}(x)=\sum_{j} \chi_{B \omega_{j}+\delta}(x-j)-\chi_{B \omega_{j}}(x-j)
$$

In the case (11) where the single-site potential is the characteristic function of a ball, this is a sum of characteristic functions of mutually disjoint annuli of width $\delta$, cf. Fig. 1 . Each of these annuli contains a ball of radius $\delta / 2$, cf. Fig. 2, and we find

$$
V_{\omega+\delta, L}(x)-V_{\omega, L}(x) \geqslant W_{\delta / 2, L}(x)
$$

where $W_{\delta / 2, L}$ is the characteristic function of a union of balls of radius $\delta / 2$ each of which is contained in a different elementary cell of the grid $\mathbb{Z}^{d}$. In the case (2) where the single-site potential is the characteristic function of a cube, an analogous argument holds.

Restrict now to $i$ such that $\lambda_{i}^{L}(\omega) \in(-\infty, b-1]$ which implies in particular 


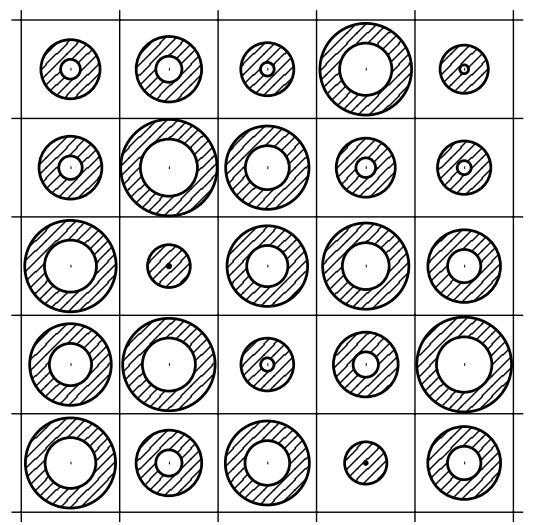

Figure 1. Support of $V_{\omega+\delta}-V_{\omega}$ in the case (11) of characteristic functions of balls as single-site potentials.

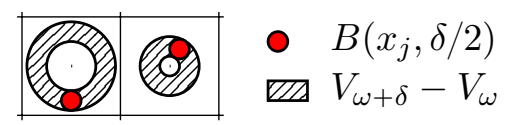

FiguRE 2. Illustration of the increments $V_{\omega+\delta}-V_{\omega}$ and the choice of the balls $B\left(x_{j}, \delta / 2\right)$ in case (11).

$\lambda_{i}^{L}(\omega+\delta) \in(-\infty, b]$. Then estimate (3) holds and we obtain via the variational characterization of eigenvalues

$$
\begin{aligned}
\lambda_{i}(\omega+\delta) & =\left\langle\phi_{i}, H_{\omega+\delta, L} \phi_{i}\right\rangle \\
& =\max _{\phi \in \operatorname{Span}\left\{\phi_{1}, \ldots, \phi_{i}\right\},\|\phi\|=1}\left\langle\phi, H_{\omega, L} \phi\right\rangle+\left\langle\phi,\left(V_{\omega+\delta, L}-V_{\omega, L}\right) \phi\right\rangle \\
& \geqslant \max _{\phi \in \operatorname{Span}\left\{\phi_{1}, \ldots, \phi_{i}\right\},\|\phi\|=1}\left\langle\phi, H_{\omega, L} \phi\right\rangle+\left\langle\phi, W_{\delta / 2, L} \phi\right\rangle \\
& \geqslant \max _{\phi \in \operatorname{Span}\left\{\phi_{1}, \ldots, \phi_{i}\right\},\|\phi\|=1}\left\langle\phi, H_{\omega, L} \phi\right\rangle+\kappa(\delta / 2)^{1 / M} \\
& \geqslant \inf _{\operatorname{dim} \mathcal{D}=i \phi \in \mathcal{D},\|\phi\|=1}\left\langle\phi, H_{\omega, L} \phi\right\rangle+\kappa(\delta / 2)^{1 / M} \\
& =\lambda_{i}(\omega)+\kappa(\delta / 2)^{1 / M} .
\end{aligned}
$$

We choose $\delta:=2\left(\frac{4 \varepsilon}{\kappa}\right)^{M}$, so that the lemma becomes

$$
\lambda_{i}(\omega+\delta) \geqslant \lambda_{i}(\omega)+4 \varepsilon .
$$

Since we required $\delta \leqslant 1 / 2-\omega_{+}$, this yields the upper bound on $\varepsilon$ from the theorem. Note in particular that this bound is smaller than $1 / 2$. Now we follow the strategy used in [5]. Let $\rho \in C^{\infty}(\mathbb{R},[-1,0])$ be a smooth, non-decreasing function such that $\rho=-1$ on $(-\infty ;-\varepsilon]$ and $\rho=0$ on $[\varepsilon ; \infty)$. We can assume $\left\|\rho^{\prime}\right\|_{\infty} \leqslant 1 / \varepsilon$. It holds that

$$
\chi_{[E-\varepsilon ; E+\varepsilon]}(x) \leqslant \rho(x-E+2 \varepsilon)-\rho(x-E-2 \varepsilon)
$$


for all $x \in \mathbb{R}$ which translates into

$$
\begin{aligned}
& \mathbb{E}\left[\operatorname{Tr}\left[\chi_{[E-\varepsilon ; E+\varepsilon]}\left(H_{\omega, L}\right)\right]\right] \\
\leqslant & \mathbb{E}\left[\operatorname{Tr}\left[\rho\left(H_{\omega, L}-E+2 \varepsilon\right)-\rho\left(H_{\omega, L}-E-2 \varepsilon\right)\right]\right] \\
= & \mathbb{E}\left[\operatorname{Tr}\left[\rho\left(H_{\omega, L}-E-2 \varepsilon+4 \varepsilon\right)-\rho\left(H_{\omega, L}-E-2 \varepsilon\right)\right]\right] .
\end{aligned}
$$

\section{Lemma 3.2.}

$$
\operatorname{Tr}\left[\rho\left(H_{\omega, L}-E-2 \varepsilon+4 \varepsilon\right)\right] \leqslant \operatorname{Tr}\left[\rho\left(H_{\omega+\delta, L}-E-2 \varepsilon\right)\right] .
$$

Proof. $\rho$ is a monotonous function, hence we have by the previous lemma

$$
\rho\left(\lambda_{i}(\omega)-E-2 \varepsilon+4 \varepsilon\right) \leqslant \rho\left(\lambda_{i}(\omega+\delta)-E-2 \varepsilon\right) .
$$

We expand the trace in eigenvalues

$$
\begin{aligned}
& \operatorname{Tr}\left[\rho\left(H_{\omega, L}-E-2 \varepsilon+4 \varepsilon\right)\right]=\sum_{k} \rho\left(\lambda_{i}(\omega)-E-2 \varepsilon+4 \varepsilon\right) \\
\leqslant & \sum_{i} \rho\left(\lambda_{i}(\omega+\delta)-E-2 \varepsilon\right)=\operatorname{Tr}\left[\rho\left(H_{\omega+\delta, L}-E-2 \varepsilon\right)\right] .
\end{aligned}
$$

Now let $\tilde{\Lambda_{L}}:=\Lambda_{L} \cap \mathbb{Z}^{d}$ and $N:=|\tilde{\Lambda}|$. The indices which affect the potential in $\Lambda_{L}$ will be enumerated by

$$
k:\{1, \ldots N\} \rightarrow \tilde{\Lambda_{L}}, \quad n \mapsto k(n) .
$$

We define functions which describe how the upper bound in (10) varies when we change one random variable $\omega_{k(n)}$ while keeping all the other random variables fixed. In order to do that we need some notation. Given $\omega \in\left[\omega_{-}, \omega_{+}\right]^{\mathbb{Z}^{d}}$, $n \in\{1, \ldots, N\}, \delta \in\left[0,1 / 2-\omega_{+}\right]$and $t \in\left[\omega_{-}, \omega_{+}\right]$, we define $\tilde{\omega}^{(n, \delta)}(t) \in\left[\omega_{-}, 1 / 2\right]^{\mathbb{Z}^{d}}$ inductively via

$$
\begin{aligned}
& \left(\tilde{\omega}^{(1, \delta)}(t)\right)_{j}:= \begin{cases}t & \text { if } j=k(1) \\
\omega_{j} & \text { else }\end{cases} \\
& \left(\tilde{\omega}^{(n, \delta)}(t)\right)_{j}:= \begin{cases}t & \text { if } j=k(n) \\
\left(\tilde{\omega}^{(n-1, \delta)}\left(\omega_{j}+\delta\right)\right)_{j} & \text { else. }\end{cases}
\end{aligned}
$$

The function $\tilde{\omega}^{(n, \delta)}:\left[\omega_{-}, 1 / 2\right] \rightarrow\left[\omega_{-}, 1 / 2\right]^{\mathbb{Z}^{d}}$ is the rank-one perturbation of $\omega$ in the $k(n)$-th coordinate with the additional requirement that all sites $k(1), \ldots, k(n-1)$ have already been blown up by $\delta$.

We define

$$
\Theta_{n}(t):=\operatorname{Tr}\left[\rho\left(H_{L}\left(\tilde{\omega}^{(n, \delta)}(t)\right)-E-2 \varepsilon\right)\right], \text { for } n=1, \ldots, N .
$$

Note that

$$
\Theta_{n}\left(\omega_{k(n)}\right)=\Theta_{n-1}\left(\omega_{k(n-1)}+\delta\right) \text { for } n=2, \ldots, N
$$

and

$$
\begin{aligned}
\Theta_{N}\left(\omega_{k(N)}+\delta\right) & =\operatorname{Tr}\left[\rho\left(H_{\omega+\delta, L}-E-2 \varepsilon\right)\right] \\
\Theta_{1}\left(\omega_{k(1)}\right) & =\operatorname{Tr}\left[\rho\left(H_{\omega, L}-E-2 \varepsilon\right)\right] .
\end{aligned}
$$


Hence we can expand the expectation of the upper bound in (10) in a telescopic sum

$$
\begin{aligned}
& \mathbb{E}\left[\operatorname{Tr}\left[\rho\left(H_{\omega+\delta, L}-E-2 \varepsilon\right)\right]-\operatorname{Tr}\left[\rho\left(H_{\omega, L}-E-2 \varepsilon\right]\right]\right. \\
= & \mathbb{E}\left[\Theta_{N}\left(\omega_{k(N)}+\delta\right)-\Theta_{1}\left(\omega_{k(1)}\right)\right] \\
= & \sum_{n=1}^{N} \mathbb{E}\left[\Theta_{n}\left(\omega_{k(n)}+\delta\right)-\Theta_{n}\left(\omega_{k(n)}\right)\right] .
\end{aligned}
$$

Since we have a product measure structure, we can apply Fubini's Theorem $\mathbb{E}\left[\Theta_{n}\left(\omega_{k(n)}+\delta\right)-\Theta_{n}\left(\omega_{k(n)}\right)\right]=\mathbb{E}\left[\int_{\omega_{-}}^{\omega_{+}} \Theta_{n}\left(\omega_{k(n)}+\delta\right)-\Theta_{n}\left(\omega_{k(n)}\right) \mathrm{d} \mu\left(\omega_{k(i)}\right)\right]$.

Note that for $t \in\left[\omega_{-}, 1 / 2\right], \Theta_{n}$ is non-decreasing and bounded. In fact, monotonicity follows from the inequality

$$
V_{L}\left(\tilde{\omega}^{(n, \delta)}\left(t_{1}\right)\right) \leqslant V_{L}\left(\tilde{\omega}^{(n, \delta)}\left(t_{2}\right)\right),
$$

whenever $t_{1} \leqslant t_{2}$ and boundedness is due to the fact that 0 and 1 provide upper and lower bounds

$$
0 \leqslant V_{L}\left(\tilde{\omega}^{(n, \delta)}(t)\right) \leqslant 1 .
$$

Lemma 3.3. Let $-\infty<\omega_{-}<\omega_{+} \leqslant+\infty$. Assume that $\mu$ is a probability distribution with bounded density $\nu_{\mu}$ and support in the interval $\left[\omega_{-}, \omega_{+}\right]$or $\left[\omega_{-}, \omega_{+}\right)$if $\omega_{+}=\infty$ and let $\Theta$ be a non-decreasing, bounded function. Then for every $\delta>0$

$$
\int_{\mathbb{R}}[\Theta(\lambda+\delta)-\Theta(\lambda)] \mathrm{d} \mu(\lambda) \leqslant\left\|\nu_{\mu}\right\|_{\infty} \cdot \delta\left[\Theta\left(\omega_{+}+\delta\right)-\Theta\left(\omega_{-}\right)\right] .
$$

Proof. We calculate

$$
\begin{aligned}
& \int_{\mathbb{R}}[\Theta(\lambda+\delta)-\Theta(\lambda)] \mathrm{d} \mu(\lambda) \\
\leqslant & \left\|\nu_{\mu}\right\|_{\infty} \int_{\omega_{-}}^{\omega_{+}}[\Theta(\lambda+\delta)-\Theta(\lambda)] \mathrm{d} \lambda=\left\|\nu_{\mu}\right\|_{\infty}\left[\int_{\omega_{-}+\delta}^{\omega_{+}+\delta} \Theta(\lambda) \mathrm{d} \lambda-\int_{\omega_{-}}^{\omega_{+}} \Theta(\lambda) \mathrm{d} \lambda\right] \\
= & \left\|\nu_{\mu}\right\|_{\infty}\left[\int_{\omega_{+}}^{\omega_{+}+\delta} \Theta(\lambda) \mathrm{d} \lambda-\int_{\omega_{-}}^{\omega_{-}+\delta} \Theta(\lambda) \mathrm{d} \lambda\right] \leqslant\left\|\nu_{\mu}\right\|_{\infty} \cdot \delta\left[\Theta\left(\omega_{+}+\delta\right)-\Theta\left(\omega_{-}\right)\right] .
\end{aligned}
$$

Thus, we find

$$
\int_{\omega_{-}}^{\omega_{+}}\left[\Theta_{i}\left(\omega_{k(i)}+\delta\right)-\Theta_{i}\left(\omega_{k(i)}\right) \mathrm{d} \mu\left(\omega_{k(i)}\right)\right] \leqslant\left\|\nu_{\mu}\right\|_{\infty} \cdot \delta\left[\Theta_{i}\left(\omega_{+}+\delta\right)-\Theta_{i}\left(\omega_{-}\right)\right]
$$

We will use the results from the previous section in the following form:

Proposition 3.4. Let $H_{0}:=H_{A}+V_{0}$ be a Schrödinger operator with a bounded potential $V_{0} \geqslant 0$, and let $H_{1}:=H_{0}+V$ for some bounded $V \geqslant 0$ with support in a cube of side length 1 . Denote the Dirichlet restrictions to $\Lambda=\Lambda_{L}$ by $H_{0}^{\Lambda}$ and $H_{1}^{\Lambda}$, respectively. There are constants $K_{1}, K_{2}$ depending only on $d$ such 
that for any smooth function $g: \mathbb{R} \rightarrow \mathbb{R}$ with derivative supported in a compact subset of $(-\infty, b]$ and the property that $g\left(H_{1}\right)-g\left(H_{0}\right)$ is trace class

$$
\operatorname{Tr}\left[g\left(H_{1}\right)-g\left(H_{0}\right)\right] \leqslant K_{1} e^{b}+K_{2}\left(\ln \left(1+\left\|g^{\prime}\right\|_{\infty}\right)^{d}\right)\left\|g^{\prime}\right\|_{1}
$$

A possible choice is $K_{1}:=2 \cdot 32^{d} \cdot(d+1) !$ and $K_{2}:=32^{d}$.

The expression $\operatorname{Tr}\left[g\left(H_{1}^{\Lambda}\right)-g\left(H_{0}^{\Lambda}\right)\right]$ is well-defined since $H_{0}^{\Lambda}$ and $H_{1}^{\Lambda}$ are both lower semibounded operators with purely discrete spectrum and only the finite set of eigenvalues in supp $g^{\prime}$ can contribute to the trace.

Proposition 3.4 implies

Lemma 3.5. Let $0<\varepsilon \leqslant \frac{1}{2}$. There is a constant $\tilde{C}$ depending only on $d$ and on $b$ such that

$$
\Theta_{n}\left(\omega_{+}+\delta\right)-\Theta_{n}\left(\omega_{-}\right) \leqslant \tilde{C}|\ln \varepsilon|^{d} .
$$

The constant $\tilde{C}$ can be chosen equal to $K_{1} e^{b}+2^{d} K_{2}$ with $K_{1}, K_{2}$ as in Proposition 3.4 .

Proof. Let $g(\cdot):=\rho_{E+2 \varepsilon}(\cdot):=\rho(\cdot-(E+2 \varepsilon))$. By our choice of $\rho, g$ has support in $(-\infty, b],\left\|g^{\prime}\right\|_{\infty} \leqslant 1 / \varepsilon$ and $\left\|g^{\prime}\right\|_{1}=1$. We define the operators

$$
\begin{aligned}
& H_{0}:=H\left(\tilde{\omega}^{(n, \delta)}\left(\omega_{-}\right)\right) \\
& H_{1}:=H\left(\tilde{\omega}^{(n, \delta)}\left(\omega_{+}+\delta\right)\right) .
\end{aligned}
$$

These are lower semibounded operators with purely discrete spectrum and since $g$ has support in $(-\infty, b]$, the difference $g\left(H_{1}\right)-g\left(H_{0}\right)$ trace class. By the previous Proposition

$\Theta_{n}\left(\omega_{+}+\delta\right)-\Theta_{n}\left(\omega_{-}\right)=\operatorname{Tr}\left[\rho_{E+2 \varepsilon}\left(H_{1}\right)-\rho_{E+2 \varepsilon}\left(H_{0}\right)\right] \leqslant K_{1} e^{b}+K_{2}(\ln (1+1 / \varepsilon))^{d}$.

We assumed $0<\varepsilon \leqslant \frac{1}{2}$, thus $1+\varepsilon \leqslant \varepsilon^{-1}$ and

$$
\ln (1+1 / \varepsilon)=\ln (1+\varepsilon)-\ln \varepsilon \leqslant-2 \ln \varepsilon=2|\ln \varepsilon|
$$

and $1 \leqslant|\ln \varepsilon| \leqslant|\ln \varepsilon|^{d}$ which proves the Lemma.

Putting everything together yields

$$
\mathbb{E}\left[\operatorname{Tr}\left[\chi_{[E-\varepsilon, E+\varepsilon]}\left(H_{\omega, L}\right)\right]\right] \leqslant\left(K_{1} e^{b}+2^{d} K_{2}\right)\left\|\nu_{\mu}\right\|_{\infty} \cdot \delta|\ln \varepsilon|^{d} L^{d} .
$$

and bearing in mind that $\delta=2 \cdot\left(\frac{4 \varepsilon}{\kappa}\right)^{1 / M}$ we obtain (4). This proves Theorem 1.3 .

\section{REFERENCES}

[1] K. Broderix, D. Hundertmark, and H. Leschke. Continuity properties of Schrödinger semigroups with magnetic fields. Rev. Math. Phys., 12(2):181-225, 2000.

[2] J.-M. Combes, P. D. Hislop, and E. Mourre. Spectral averaging, perturbation of singular spectra, and localization. Trans. Amer. Math. Soc., 348(12):4883-4894, 1996.

[3] J.-M. Combes, P. D. Hislop, and S. Nakamura. The $L^{p}$-theory of the spectral shift function, the Wegner estimate, and the integrated density of states for some random Schrödinger operators. Commun. Math. Phys., 70(218):113-130, 2001.

[4] L. C. Evans. Partial differential equations, volume 19 of Graduate Studies in Mathematics. American Mathematical Society, Providence, RI, 1998. 
[5] D. Hundertmark, R. Killip, S. Nakamura, P. Stollmann, and I. Veselić. Bounds on the spectral shift function and the density of states. Comm. Math. Phys., 262(2):489-503, 2006. ArXiv.org/math-ph/0412078.

[6] D. Hundertmark and B. Simon. An optimal $L^{p}$-bound on the Krein spectral shift function. J. Anal. Math., 87:199-208, 2002. http://www.ma.utexas.edu/mp_arc/c/00/00-370.ps.gz.

[7] D. Hundertmark and B. Simon. A diamagnetic inequality for semigroup differences $J$. Reine Angew. Math., 571:107-130, 2004

[8] W. Kirsch and I. Veselić. Lifshitz tails for a class of Schrödinger operators with random breather-type potential. Lett. Math. Phys., 94(1):27-39, 2010.

[9] A. Klein. Unique continuation principle for spectral projections of Schrödinger operators and optimal Wegner estimates for non-ergodic random schrödinger operators. Comm. Math. Phys., 323(3):1229-1246, 2013.

[10] F. Klopp, M. Loss, S. Nakamura, and G. Stolz. Localization for the random displacement model. Duke Math. J., 161(4):587-621, 2012.

[11] H.- Leinfelder. Gauge invariance of Schrödinger operators and related spectral properties. J. Operator Theory, 9:163-179, 1983.

[12] I. Nakić, M. Täufer, M. Tautenhahn, and I. Veselić. Scale-free uncertainty principles and applications. Working paper, Technische Universität Chemnitz.

[13] I. Nakić, M. Täufer, M. Tautenhahn, and I. Veselić. Scale-free uncertainty principles and Wegner estimates for random breather potentials. ArXiv.org/1410.5273. To appear in Comptes Rendus Mathematique.

[14] C. Rojas-Molina and I. Veselić. Scale-free unique continuation estimates and applications to random Schrödinger operators. Comm. Math. Phys., 320(1):245-274, 2013. http://ArXiv.org/1110.4652.

[15] C. Schumacher and I. Veselić. Lifschitz tails for random breather potentials. Working paper, Technische Universität Chemnitz.

[16] B. Simon. Functional integration and quantum physics Pure and Applied Mathematics, 86, Academic Press Inc. [Harcourt Brace Jovanovich Publishers], New York, 1979.

[17] I. Veselić. Lifshitz asymptotics for Hamiltonians monotone in the randomness. Oberwolfach Rep., 4(1):380-382, 2007. ArXiv.org/0708.0487.

[18] I. Veselić. Existence and regularity properties of the integrated density of states of random Schrödinger Operators, volume Vol. 1917 of Lecture Notes in Mathematics. SpringerVerlag, 2008.

[19] D. Yafaev. Mathematical Scattering Theory. Translations of Mathematical Monographs, 105. American Mathematical Society, Providence, Rhode Island, 1992. [Russian original: Izdatel' stvo Sankt-Peterburgskogo Universiteta, St. Petersburg, 1994].

Fakultät für Mathematik, TU-Chemnitz, Germany

E-mail address: matthias.taeufer@mathematik.tu-chemnitz.de

$U R L:$ www.tu-chemnitz.de/mathematik/stochastik 